PROCEEDINGS OF THE

AMERICAN MATHEMATICAL SOCIETY

Volume 132, Number 2, Pages 443-446

S 0002-9939(03)07215-0

Article electronically published on August 7, 2003

\title{
SPECTRALLY BOUNDED OPERATORS ON SIMPLE $C^{*}$-ALGEBRAS
}

\author{
MARTIN MATHIEU \\ (Communicated by David R. Larson)
}

\begin{abstract}
A linear mapping $T$ from a subspace $E$ of a Banach algebra into another Banach algebra is called spectrally bounded if there is a constant $M \geq 0$ such that $r(T x) \leq M r(x)$ for all $x \in E$, where $r(\cdot)$ denotes the spectral radius. We prove that every spectrally bounded unital operator from a unital purely infinite simple $C^{*}$-algebra onto a unital semisimple Banach algebra is a Jordan epimorphism.
\end{abstract}

\section{INTRODUCTION AND MAIN RESULT}

A simple $C^{*}$-algebra $A$ is said to be purely infinite if every nonzero hereditary $C^{*}$-subalgebra is infinite. In particular, every nonzero projection has to be infinite. Zhang showed in 13 that every purely infinite simple $C^{*}$-algebra has real rank zero, that is, the selfadjoint elements with finite spectrum are dense in the selfadjoint part of the $C^{*}$-algebra. Whether the converse holds, i.e., an infinite simple $C^{*}$-algebra with real rank zero has to be purely infinite, remains an open problem. By Rørdam's example 12], the assumption of real rank zero is crucial here.

Let $A$ be a unital $C^{*}$-algebra, and let $B$ be a unital semisimple Banach algebra. A linear mapping $T: A \rightarrow B$ is called a Jordan epimorphism if it is surjective and $T\left(x^{2}\right)=(T x)^{2}$ for all $x \in A$. It is well known that every Jordan epimorphism is unital, that is, $T 1=1$, and preserves invertibility. Denoting by $r(x)$ the spectral radius of an element $x$, it thus follows that $T$ satisfies the estimate $r(T x) \leq r(x)$ for all $x \in A$. More generally, let $E \subseteq A$ be a subspace of $A$. Then $T: E \rightarrow B$ is said to be spectrally bounded if there exists a constant $M \geq 0$ such that $r(T x) \leq M r(x)$ for all $x \in A$. This concept has proven to be very useful in automatic continuity theory; see, for instance, [1]. A number of basic properties of spectrally bounded operators are established in [8].

The following spectral characterization of Jordan epimorphisms was obtained in [9, Theorem 3.6].

Received by the editors September 30, 2002.

2000 Mathematics Subject Classification. Primary 47B48; Secondary 46L05, 47A65, 17C65.

Key words and phrases. Spectrally bounded operators, Jordan homomorphisms, purely infinite simple $C^{*}$-algebras.

This paper was written during a visit to the Departamento de Análisis Matemático de la Universidad de Granada, Granada, Spain. The author gratefully acknowledges the generous hospitality extended to him by his colleagues there. The paper is part of the research carried out in the EC network Analysis and Operators (HPRN-CT-2000-00116).

(C)2003 American Mathematical Society 
Theorem A. Let $T: A \rightarrow B$ be a unital surjective spectrally bounded operator from a properly infinite von Neumann algebra A onto a unital semisimple Banach algebra $B$. Then $T$ is a Jordan epimorphism.

The hypothesis on the domain turns out to be essential, since on a commutative von Neumann algebra every bounded operator is spectrally bounded; hence the theorem is bound to fail in the finite case.

In the present note we take a first step to extend Theorem A appropriately to the setting of $C^{*}$-algebras. Since the center is not easy to control, we restrict our attention to simple $C^{*}$-algebras. Since an abundance of projections is needed (see Lemma 1 below), we assume that the $C^{*}$-algebra has real rank zero. Also, since the existence of traces provides an obstruction, we confine ourselves to infinite $C^{*}$-algebras. That is why, in this paper, the framework of unital purely infinite simple $C^{*}$-algebras is chosen.

With this background in mind, we will prove the following theorem, which is the exact analogue of Theorem A.

Theorem B. Let $T: A \rightarrow B$ be a unital surjective spectrally bounded operator from a unital purely infinite simple $C^{*}$-algebra $A$ onto a unital semisimple Banach algebra B. Then $T$ is a Jordan epimorphism.

\section{Proof of the MAIN RESUlt}

The first lemma will allow us to do the final step in the proof of Theorem B. It was obtained for von Neumann algebras in [9, Lemma 2.1]. Since the argument relies only on the fact that every selfadjoint element can be approximated by finite linear combinations of orthogonal projections, and this holds in every $C^{*}$-algebra of real rank zero by [2], the proof takes over without problems and is hence omitted here.

Lemma 1. Let $T: A \rightarrow B$ be a bounded linear operator from a $C^{*}$-algebra $A$ of real rank zero into a Banach algebra $B$ sending projections in $A$ to idempotents in B. Then $T$ is a Jordan homomorphism.

The next lemma is a special case of [9, Lemma 3.1]. From a purely spectral hypothesis we derive a strong algebraic property of the operator $T$.

Lemma 2. Let $T: A \rightarrow B$ be a spectrally bounded operator from a $C^{*}$-algebra $A$ onto a semisimple Banach algebra B. Suppose that $x \in A$ satisfies $x^{2}=0$. Then $(T x)^{2}=0$.

In order to put Lemma 2 into action we need a good supply with elements of square zero in the domain. This is provided by the following result.

Lemma 3. Let $A$ be a unital purely infinite simple $C^{*}$-algebra, and let $p$ be a nonzero projection in $A$. Then each element in $p A p$ is a finite sum of elements in pAp with square zero.

Proof. Since every infinite projection in $A$ is properly infinite by [3, Proposition 2.2], there exist orthogonal subprojections $p_{1}, p_{2}$ of $p$ such that $p_{1} \sim p_{2} \sim p$. Thus [5] Theorem 2.1] entails that every element in $p A p$ is the sum of 10 commutators (see also [11]). Put $p_{3}=p-\left(p_{1}+p_{2}\right)$ and note that $p_{3}$ is a proper projection in $p A p$ and orthogonal to $p_{1}$ and $p_{2}$. Since $p-p_{3}$ is infinite and $A$ is simple, it follows that $p_{3} \precsim p-p_{3}$ [4. Lemma V.5.4], and it is clear that $p_{i} \precsim p-p_{i}$ for $i=1,2$. 
Therefore, the assumptions of [6, Theorem 3.5] are satisfied, and it follows that every commutator in $p A p$ is the sum of 13 elements in $p A p$ of square zero. As a result, for each $x \in p A p$, there are at most 130 elements in $p A p$ with square zero whose sum is $x$.

To complete the preparations for the proof of Theorem B, we note the following well-known automatic continuity result, which is a direct consequence of, e.g., [1] Lemma A].

Lemma 4. Let $T: A \rightarrow B$ be a spectrally bounded operator from a $C^{*}$-algebra $A$ onto a semisimple Banach algebra $B$. Then $T$ is bounded.

Proof of Theorem B. By [13], $A$ has real rank zero; so in view of Lemmas 1 and 4 we only need to show that $T$ maps every projection in $A$ onto an idempotent in $B$.

Let $p$ be a nonzero projection in $A$ such that $q=1-p$ is nonzero as well. If $a \in p A p$ and $b \in q A q$, by Lemma 3 , there are finitely many $a_{i} \in p A p, b_{j} \in q A q$ such that $a=\sum_{i} a_{i}, b=\sum_{j} b_{j}$, and $a_{i}^{2}=b_{j}^{2}=0$ for all $i, j$. We claim that

$$
(T a)(T b)+(T b)(T a)=0 .
$$

Since $\left(a_{i}+b_{j}\right)^{2}=0$ for all $i, j$, Lemma 2 entails that $\left(T\left(a_{i}+b_{j}\right)\right)^{2}=0$ for all $i, j$. On the other hand,

$\left(T\left(a_{i}+b_{j}\right)\right)^{2}=\left(T a_{i}\right)^{2}+\left(T a_{i}\right)\left(T b_{j}\right)+\left(T b_{j}\right)\left(T a_{i}\right)+\left(T b_{j}\right)^{2}=\left(T a_{i}\right)\left(T b_{j}\right)+\left(T b_{j}\right)\left(T a_{i}\right)$, wherefore $\left(T a_{i}\right)\left(T b_{j}\right)+\left(T b_{j}\right)\left(T a_{i}\right)=0$ for all $i, j$. Summing over all indices yields the claim.

Applying (1) to $a=p$ and $b=1-p$ yields

$$
2\left(T p-(T p)^{2}\right)=(T p)(1-T p)+(1-T p)(T p)=0,
$$

since $T 1=1$. Consequently, $T p$ is idempotent.

\section{AN OUTLOOK}

It appears that the main obstruction to an extension of Theorem $\mathrm{B}$ to finite (simple) $C^{*}$-algebras is the existence of bounded traces. Indeed, if $A$ is a finitedimensional simple $C^{*}$-algebra, every surjective unital spectrally bounded operator from $A$ into itself is a linear combination of the canonical trace on $A$ and a Jordan automorphism of $A$. In [7] we showed that a unital spectrally bounded operator from a unital $C^{*}$-algebra into its center necessarily is a bounded trace (though possibly not positive). Therefore it seems to be conceivable that a splitting of a spectrally bounded operator whose values lie in a $C^{*}$-algebra that has a nonzero finite trace into a superposition of a bounded trace and a Jordan homomorphism is possible. We have not been able to establish this yet.

Call a linear mapping $T$ between $C^{*}$-algebras a spectral isometry if $r(T x)=r(x)$ for all $x$ in the domain. In 8 we raised the following question: Is every unital surjective spectral isometry between unital $C^{*}$-algebras necessarily a Jordan isomorphism? We summarize the results known until now including the consequences of Theorems A and B above in the following result.

Theorem C. Let $T: A \rightarrow B$ be a unital surjective spectral isometry between the unital $C^{*}$-algebras $A$ and $B$. Then $T$ is a Jordan isomorphism provided $A$ or $B$ belong to one of the following classes of $C^{*}$-algebras: 
(1) properly infinite von Neumann algebras;

(2) unital purely infinite simple $C^{*}$-algebras;

(3) commutative $C^{*}$-algebras;

(4) finite-dimensional $C^{*}$-algebras.

Proof. Note at first that $T$ is injective, by [8, Proposition 4.2]. Therefore, $T^{-1}: B \rightarrow$ $A$ is a unital spectral isometry as well. Statements (1) and (2) thus follow by applying Theorem A and Theorem B, respectively, to $T$ or to $T^{-1}$.

Suppose that $A$ is commutative. By [ 8 Corollary 4.4], $T$ is an algebra isomorphism from $A$ onto $Z(B)$, the center of $B$. Let $S=T \circ T^{-1}$; this is a unital bijective spectral isometry from $B$ onto $Z(B)$. By [7 Lemma 2.1], $S(x y)=S(y x)$ for all $x, y \in B$. Since $S$ is injective, $B$ is commutative and the result follows. The other case in statement (3) is obtained by considering $T^{-1}$ instead of $T$.

Statement (4) is a consequence of joint work with A. R. Sourour, which appears in [10].

\section{REFERENCES}

1. B. Aupetit and M. Mathieu, The continuity of Lie homomorphisms, Studia Math. 138 (2000), 193-199. MR 2001a:46050

2. L. G. Brown and G. K. Pedersen, $C^{*}$-algebras of real rank zero, J. Funct. Anal. 99 (1991), 131-149. MR 92m:46086

3. J. Cuntz, The structure of multiplication and addition in simple $C^{*}$-algebras, Math. Scand. 40 (1977), 215-233. MR 58:17862

4. K. R. Davidson, $C^{*}$-algebras by example, Fields Inst. Monographs 6, Amer. Math. Soc., Providence, RI, 1996. MR 97i:46095

5. T. Fack, Finite sums of commutators in $C^{*}$-algebras, Ann. Inst. Fourier, Grenoble 32 (1982), 129-137. MR 83g:46051

6. L. W. Marcoux, On the linear span of the projections in certain simple $C^{*}$-algebras, Indiana Univ. Math. J. 51 (2002), 753-771.

7. M. Mathieu, Spectrally bounded traces on $C^{*}$-algebras, Bull. Austral. Math. Soc. 68 (2003), 169-173.

8. M. Mathieu and G. J. Schick, First results on spectrally bounded operators, Studia Math. 152 (2002), 187-199. MR 2003e:47066

9. M. Mathieu and G. J. Schick, Spectrally bounded operators from von Neumann algebras, in press, J. Operator Theory (2003).

10. M. Mathieu and A. R. Sourour, Hereditary properties of spectral isometries, Archiv Math. (Basel), to appear.

11. C. Pop, Finite sums of commutators, Proc. Amer. Math. Soc. 130 (2002), 3039-3041. MR 2003c: 46070

12. M. Rørdam, A simple $C^{*}$-algebra with a finite and an infinite projection, preprint.

13. S. Zhang, A property of purely infinite simple $C^{*}$-algebras, Proc. Amer. Math. Soc. 109 (1990), 717-720. MR 90k:46134

Department of Pure Mathematics, Queen's University Belfast, Belfast BT7 1NN, NORTHERN IRELAND

E-mail address: m.m@qub.ac.uk 